Research Article

\title{
Determination of Cyclopropane Fatty Acids in Food of Animal Origin by ${ }^{1} \mathrm{H}$ NMR
}

\author{
Veronica Lolli (D), Angela Marseglia (iD, Gerardo Palla, Emanuela Zanardi, \\ and Augusta Caligiani \\ Department of Food and Drug, University of Parma, Parco Area delle Scienze 27A, 43124 Parma, Italy \\ Correspondence should be addressed to Augusta Caligiani; augusta.caligiani@unipr.it
}

Received 31 July 2017; Revised 11 October 2017; Accepted 12 February 2018; Published 1 April 2018

Academic Editor: Mercedes G. Lopez

Copyright ( 2018 Veronica Lolli et al. This is an open access article distributed under the Creative Commons Attribution License, which permits unrestricted use, distribution, and reproduction in any medium, provided the original work is properly cited.

\begin{abstract}
Cyclopropane fatty acids (CPFAs) are unusual fatty acids of microbial origin, recently detected in milk and dairy products. CPFAs have been demonstrated to be interesting molecular markers for authentication of dairy products obtained without ensiled feeds. Moreover, they can also be recognized as a new secondary component of human diet. Information is lacking on the presence of cyclic fatty acids in other food sources. Cyclopropane fatty acids have been detected by GC-MS analysis in cheese and other animal fats in concentration ranging from 200 to $1000 \mathrm{mg} / \mathrm{kg}$ fat, but in some cases, the complex fatty acid profile and the possible presence of interfering peaks make the separation not straightforward and the quantification uneasy. Therefore, a new reliable ${ }^{1}$ H NMR method was developed to detect and measure CPFA content in different foods of animal origin, based on the detection of the characteristic signals of cyclopropane ring. The ${ }^{1} \mathrm{H}$ NMR $(600 \mathrm{MHz})$ method showed detection limits comparable with those of full scan GC-MS, and it allowed the identification and quantitation of the cyclopropane fatty acids in different foods.
\end{abstract}

\section{Introduction}

Cyclopropane fatty acids are unusual fatty acids found in microorganisms, both Gram-negative and Gram-positive, and seed oils of some tropical plants and protozoa $[1,2]$. The bacterial production of cyclopropane ring is related to changes in the membrane fatty acids composition and represents one of the most important adaptive microbial responses that favours the stress tolerance of several bacteria, such as Lactobacillus helveticus, L. bulgaricus, L. acidophilus, and L. sanfranciscensis [1].

In plants, CPFAs are usually minor components, where cyclopropene fatty acids are the most abundant. They are present in Malvaceae, Sterculiaceae, and Sapindaceae, representing a significant component of Litchi chinensis and Sterculia foetida seed oils, principally sterculic acid [2].

Recently, we identified by GC-MS the presence of CPFAs (dihydrosterculic and lactobacillic acids, Figure 1) in milk and dairy products $[3,4]$ and more recently in meat (unpublished results).

Due to the undoubtable importance of these foodstuffs in human diet, it appears clear that a deep investigation on the dietary intake of these fatty acids and their effects on humans gains importance.

CPFAs have been also recently identified in human serum and adipose tissue [5], suggesting that they are absorbed as the other fatty acids and can exert physiological effects. Moreover, CPFAs are minor fatty acids but their presence in milk fat is in the hundred-ppm order [4], so their dietary intake may be not negligible.

CPFAs (mainly dihydrosterculic acid) also play an important role in food authentication: in fact, they were discovered in milk and dairy products from cows fed with silages, and their determination has been demonstrated to be a powerful tool for the authentication of Protected Denomination of Origin (PDO) cheeses, such as Parmigiano Reggiano, where the use of silages in cow feeding is forbidden [6]. In this context, "Consorzio del Formaggio Parmigiano Reggiano" has proposed a modification on the Production Specification Rules, including the determination of CPFAs among the official controls (UNI 11650).

Therefore, CPFAs represent an almost completely new field of research in food lipids and it is important to develop different methods of detection and quantification, in view of 


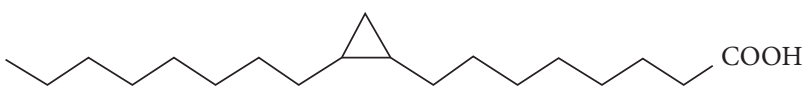

Dihydrosterculic acid

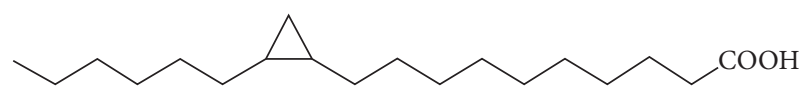

Lactobacillic acid

FIGURE 1: Main cyclopropane fatty acids detected in dairy products.

an expected growing body of research, both in food characterization/authentication and in food safety aspects.

Gas chromatography methods currently dominate the literature for the determination of main and secondary fatty acids in foods [7-9], and we previously applied this technique for the qualitative and quantitative determination of CPFAs in milk and dairy products $[3,4,6]$. However, gas chromatography analysis requires timeconsuming sample derivatization with the risk of interfering by-products and use of large amount of solvents [10]. Moreover, in the particular case of fat from animal origin, the extreme complexity of fatty acid profile makes the separation and quantification of minor fatty acids a challenging issue. For example, more than 400 different fatty acids were detected in milk [11]. In the case of cyclopropane fatty acids, we obtained its separation in cheese fat by using apolar capillary column [6]; however, this column is not suitable for the optimal separation of fatty acids, so it is possible that changing the food matrix interferences occur. It is also possible that other cyclopropane fatty acids were present but undetectable because they were overlapped by the most abundant fatty acid signals. So, it is important to have an alternative method to confirm the cyclopropane ring presence and possibly to correctly quantify CPFAs. Moreover, the development of a rapid method that provides the necessary analytical information with minimal sample preparation would be advantageous. NMR spectroscopy is one such analytical tool that avoids sample derivatization and offers the benefit of short data acquisition times.

Nuclear magnetic resonance spectroscopy has started to represent an interesting tool to analyse biofluids and food and beverages, and in the case of lipids, it represents a reliable and fast alternative to traditional methods such as gas chromatography. This was due to the advantages of this technique as the simplicity of the sample preparation (usually it only requires the fat dissolution in deuterated chloroform) and measurement procedures, the instrumental stability, the increase of sensitivity, and modern pulse sequences, with simultaneous suppression of big signals [12].

For these reasons, the use of NMR spectroscopy has established a significant role in the analysis of lipids [13]. Several studies consider the analysis by ${ }^{1} \mathrm{H}$ NMR of triacylglycerol composition as a useful tool for both triglyceride quantitation and sample classification [14]. Minor fatty acids were also object of investigation by NMR, especially conjugated linoleic acids (CLAs) [15].
NMR could represent an ideal method to detect CPFAs due to the characteristic signals of the protons of the cyclopropane unit between -0.30 and -0.35 ppm [16], which permit their detection in a zone of ${ }^{1} \mathrm{H}$ NMR spectrum practically free from other signals. This highly shielded position of cyclopropane resonance is conventionally explained by the anisotropy of the $\mathrm{C}-\mathrm{C}$ bond, just opposite to $\mathrm{CH}_{2}$ group in a three-membered ring, or by an aromaticlike ring current involving the six electrons in the three $\mathrm{C}-\mathrm{C}$ bonds ( $\sigma$ aromaticity) that shields cyclopropane protons [17].

Therefore, with the aim to investigate on the presence of CPFAs in foods, we developed a new fast and reliable quantitative ${ }^{1} \mathrm{H}$ NMR method, to be used as alternative to gas chromatographic methods and to confirm the presence of CPFAs in foods.

\section{Experimental}

2.1. Materials. Methanol, $n$-hexane, dichloromethane, trimethylchlorosilane, hexamethyldisilazane, 1-decanol, sodium sulphate anhydrous, sodium carbonate, deuterated chloroform, and tetracosane were from Sigma-Aldrich (Saint Louis, MO, USA), and hydrochloric acid and potassium hydroxide pellets were from Carlo Erba (Milan, Italy). Dihydrosterculic acid methyl ester was from Abcam (Cambridge).

All the solvents, standards, and reagents were of analytical grade.

Cheese, meat samples from several species animals, cured meat, and commercial fish were analysed for the content of cyclopropane fatty acids. Most of them were purchased from the market (Parma, Italy). Samples of cheese and meat produced without ensiled feeds were kindly provided from Parmigiano Reggiano Cheese Consortium and Prof. Riccardo Bozzi of the University of Florence, respectively.

2.2. Fat Extraction. Lipid extraction following the Folch method [18] was performed. $10 \mathrm{~g}$ of sample was homogenized with $75 \mathrm{~mL}$ of dichloromethane : methanol $(2: 1, \mathrm{v} / \mathrm{v})$. The mixture was centrifuged $(10 \mathrm{~min}, 3000 \mathrm{rpm})$ and filtered. This procedure was repeated three times. The three filtrates were transferred to a graduate cylinder, and a volume of about $50 \mathrm{~mL} \mathrm{KCl} 0.88 \%$ in distilled water was added. The mixture was shaken vigorously. The final biphasic system was decanted, and the upper aqueous phase was eliminated. The lower organic phase was filtered through anhydrous sodium sulphate and collected. Lipid content was then recovered after solvent was evaporated with a rotary evaporator under vacuum.

\section{3. ${ }^{1} \mathrm{H}$ NMR Analysis}

2.3.1. Synthesis of Internal Standard Trimethylsilyl Decanol (TMSD). $0.2 \mathrm{~mL}$ of 1-decanol, $0.3 \mathrm{~mL}$ of trimethylchlorosilane, and $0.6 \mathrm{~mL}$ of hexamethyldisyiazane were mixed in a screw cap septum vial. Mixture reacted for $1 \mathrm{~h}$ at $60^{\circ} \mathrm{C}$, neutralized 
with sodium carbonate, and then dried with anhydrous sodium sulphate. Reaction mixture was diluted with $1 \mathrm{~mL}$ of hexane, filtered, taken to dryness in a rotary evaporator, and the residue weighed. Purity of trimethylsilyl decanol (TMSD) was confirmed by ${ }^{1} \mathrm{H}$ NMR and by GC-MS analysis in the conditions reported in Section 2.4.

\subsubsection{Preparation of CPFA and TMSD Standard Solutions.} Appropriate amounts of trimethylsilyl decanol (TMSD, internal standard) and CPFAs were weighed and added separately to $\mathrm{CDCl}_{3}(10 \mathrm{~mL})$ to yield two final stock solutions of about $500 \mathrm{mg} / \mathrm{L}$ each.

Adequate amounts of CPFA and TMSD stock solutions were transferred in $5 \mathrm{~mm} \mathrm{NMR}$ tubes and taken to the final volume of $1 \mathrm{~mL}$ with $\mathrm{CDCl}_{3}$ to obtain working solutions at $100,50,25$, and $5 \mu \mathrm{g} / \mathrm{mL}$ of CPFAs, all containing $10 \mu \mathrm{g} / \mathrm{mL}$ of TMSD.

2.3.3. Preparation of Spiked Samples. $100 \mathrm{mg}$ of meat fat (chicken) and cheese fat (Parmigiano Reggiano) both negative to CPFAs were spiked with the appropriate amount of CPFA and TMSD solutions and taken to the volume of $1 \mathrm{~mL}$ of $\mathrm{CDCl}_{3}$ to obtain the same final concentrations reported above for standard solutions.

2.3.4. ${ }^{1} \mathrm{H}$ NMR Acquisition. $100 \mathrm{mg}$ of fat was dissolved in $1 \mathrm{~mL}$ of $\mathrm{CDCl}_{3}$ containing $0.01 \mathrm{mg}$ of TMSD as internal standard. ${ }^{1} \mathrm{H}$ NMR spectra were recorded on a Varian INOVA-600 MHz spectrometer (Varian, Palo Alto, CA, USA), equipped with a $5 \mathrm{~mm}$ triple resonance inverse probe. Data were collected at $298 \mathrm{~K}$, with $32 \mathrm{~K}$ complex points, using a $90^{\circ}$ pulse length. 1024 scans were acquired with an acquisition time of $1.707 \mathrm{~s}$ and a recycle delay of $2 \mathrm{~s}$. Presaturation of the fatty acids $-\mathrm{CH}_{2}-$ signal $(1.25 \mathrm{ppm})$ was performed in order to assure a correct digitization of small signals as CPFAs. The NMR spectra were processed by MestReC software 6.0.2 (Santiago de Compostela, Spain, EU): spectra were Fourier transformed with FT size of $64 \mathrm{k}$ and $1 \mathrm{~Hz}$ line-broadening factor, manually phased and carefully baseline corrected, and referenced to the chloroform signal $(7.26 \mathrm{ppm})$. Baseline correction was further manually optimized in the zone of interest (from $-1 \mathrm{ppm}$ to $0.7 \mathrm{ppm})$.

2.3.5. Quantitative Analysis. CPFA concentrations were obtained by integrating the peak area of the ${ }^{1} \mathrm{H}$ NMR signal at $-0.35 \mathrm{ppm}$ and the methyl signal of the trimethylsilyl group of the internal standard (TMSD) at $0.1 \mathrm{ppm}$.

The CPFA integral was converted in mass value $(\mathrm{mg})$ according to the following formula, as previously reported [19]:

$$
\mathrm{ACPFA} \times \frac{\mathrm{EWCPFA}}{\mathrm{mg} \text { CPFA }}=\mathrm{ATMSD} \times \frac{\mathrm{EWTMSD}}{\mathrm{mg} \text { TMSD }},
$$

where $\mathrm{ACPFA}=$ spectral area of CPFA, $\mathrm{ATMSD}=$ spectral area of internal standard, EWCPFA = equivalent weight of the analyte, EWTMSD = equivalent weight of internal standard, and $\mathrm{EW}=$ (molecular weight/number of hydrogens in the signal).

Absolute amount of CPFAs obtained was finally expressed as $\mathrm{mg} / \mathrm{kg}$ of fat.

2.3.6. Linearity and Limit of Detection and Quantification. The limit of detection (LOD) and the limit of quantification (LOQ) were calculated utilizing the $S / N$ ratio methods, based on the determination of the peak-to-peak noise [20]. LOD and LOQ were, therefore, calculated as the concentrations of CPFAs producing a recognizable peak with a signal-to-noise ratio of, respectively, 3.3 and 10 . LOD and LOQ were determined both in pure standard solution and in a sample of meat fat negative to CPFAs spiked with different concentrations of CPFAs.

2.3.7. Accuracy, Precision, and Recovery of the Method. The accuracy of the CPFA recovery was determined by assaying samples with known concentrations of CPFAs, both as pure compounds and as spiked matrix. The precision was expressed as coefficient of variation (CV\%). Recovery of analytes was determined by spiking sample of fat free from CPFAs with pure dihydrosterculic acid.

2.4. Gas Chromatographic Analysis. GC-MS quantitative analysis was performed as previously reported [6]. Briefly, $200 \mathrm{mg}$ of fat was dissolved in hexane $(5 \mathrm{~mL})$ and mixed for 1 min with $0.2 \mathrm{~mL}$ of $\mathrm{KOH} 10 \%$ (Carlo Erba, Milan, Italy) in methanol. After phase separation, the superior organic phase was added to internal standard (tetracosane) and injected $(1 \mu \mathrm{L}$, split mode) on an Agilent Technologies $6890 \mathrm{~N}$ gas chromatograph (Agilent Technologies, Palo Alto, CA, USA) coupled to an Agilent Technologies 5973 mass spectrometer (Agilent Technologies, Palo Alto, CA, USA). A low-polarity capillary column (SLB-5ms, Supelco, Bellafonte, USA) was used. The chromatogram was recorded in the scan mode $(40-500 \mathrm{~m} / z)$ with a programmed temperature from $60^{\circ} \mathrm{C}$ to $280^{\circ} \mathrm{C}$.

\section{Results and Discussion}

3.1. CPFA Signal Detection. Figure 2 depicts the characteristic upfield zone of ${ }^{1} \mathrm{H}$ NMR spectrum of dihydrosterculic acid and TMCD. ${ }^{1} \mathrm{H}$ NMR shows two individual peaks at -0.35 and $0.60 \mathrm{ppm}$ for the methylene protons of the cyclopropane ring. Assignments were previously made by Knothe [16] with the aid of 2D correlations: the upfield signal is assigned to the cis-proton and the downfield signal to the trans-proton. The two methine protons of the cyclopropane ring are located at $0.68 \mathrm{ppm}$. The other CPFA proton signals are located at lower fields; for example, the four protons in alpha position with respect to the cyclopropane ring display a distinct shift at $1.17 \mathrm{ppm}$ and the signal of the other two protons is observed at $1.40 \mathrm{ppm}$, within the broad methylene peak [16]. Among all these specific signals, the cis-methylene proton of the cyclopropane ring can be easily assigned and used for quantification 


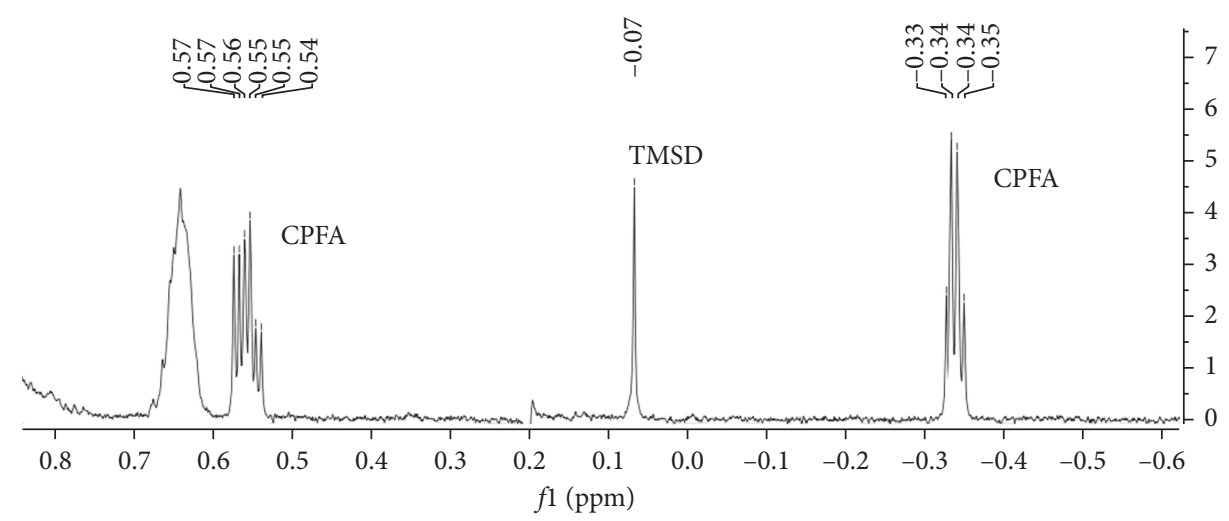

FIgure 2: ${ }^{1} \mathrm{H}$ NMR spectrum $\left(600 \mathrm{MHz}, \mathrm{CDCl}_{3}\right.$ ) of dihydrosterculic acid standard and trimethylsilyl decanol (TMSD) in the very upfield region of the spectrum. The CPFA signal at $-0.34 \mathrm{ppm}$ and the TMSD signal at $0.07 \mathrm{ppm}$ were selected for quantification.

because it does not overlap with any other signal of fatty acids that could be observed in a complex food lipid ${ }^{1} \mathrm{H}$ NMR spectrum.

Because the integral of a given peak in a ${ }^{1} \mathrm{H}$ NMR spectrum is directly proportional to a corresponding number of resonant nuclei, peak areas of CPFA cis-methylene proton can be compared with the peak area of TMSD trimethylsilyl group close to $0.1 \mathrm{ppm}$ and used for the determination of total CPFA content. This internal standard was specifically synthetized starting from a medium chain linear alcohol as decanol because it is not volatile, soluble in apolar solvents, and its trimethylsilyl group gives a singlet close to the CPFA selected signal. Tetramethylsilane (TMS) was commercially available but it cannot be used as quantitative internal standard due to its volatility. The synthesis of TMSD was quick and simple with a high yield (about $80 \%$ ). The purity was determined by GC-MS and ${ }^{1} \mathrm{H}$ NMR (data not shown).

${ }^{1} \mathrm{H}$ NMR spectra of food fats are characterized by a dominating fatty acid methylene group peak at $1.2 \mathrm{ppm}$ that is many orders of magnitude greater than those of the component of interest. This causes a number of problems: first of all, it prevents a correct digitization of small signals, hampering their observation and quantification, but the tail of this large signal can also determine a distortion of the baseline in the zone of CPFA chemical shifts. Therefore, a suppression of this signal was performed during spectra acquisition.

3.2. Quality Parameters of ${ }^{1} H$ NMR Analysis. The quantitative ${ }^{1} \mathrm{H}$ NMR method was developed with the aim to determine CPFA concentration in a broad range of food fats, in particular in fats of animal origin (dairy products, meat, and fish). As a first step, the method was subjected to validation in terms of precision, accuracy, linearity, detection, and quantitation limits, following recommendations of the International Conference on Harmonization (ICH 2005: Validation of analytical procedures: text and methodology. Harmonized tripartite guideline, Q2, R1). The validation tests were performed on pure solutions of CPFA (dihydrosterculic acid) and on cheese and meat matrices naturally free from CPFAs, spiked with dihydrosterculic acid as reported in Experimental.

To determinate accuracy and precision, solutions containing weighed amount of CPFAs were analysed by ${ }^{1} \mathrm{H}$ NMR in the experimental conditions previously reported. Measured results for the standard solutions were in agreement with the amounts weighed in the range of concentrations of $0.005-0.1 \mathrm{mg} / \mathrm{mL}$ of CPFA, a range that corresponds with the final intube concentration of the analytes in real samples of fat. The linearity was demonstrated in the same range (Figure 3 ). The limit of detection and the limit of quantification were calculated utilizing the $S / N$ ratio method described above. LOD and LOQ were calculated in pure standard solutions. The instrumental quantification limit for CPFA standard (LOQ, signal to noise ratio higher than 10) in the experimental conditions reported was about $0.01 \mathrm{mg} / \mathrm{mL}$ while the limit of detection (LOD) was obtained at $0.0025 \mathrm{mg} / \mathrm{mL}(S / N$ ratio 4$)$. The ${ }^{1} \mathrm{H}$ NMR $(600 \mathrm{MHz})$ method developed showed detection limits in pure standard solutions comparable with those generally achieved by full scan GC-MS. Coefficients of variation $(\mathrm{CV} \%)$ for three replicate measurements of each standard concentration were lower than 3\%, indicating a good precision of the method.

Linearity, LOD, and LOQ were also calculated in two different matrices, cheese and chicken fat. The samples chosen for spiking were previously analysed by GC-MS and ${ }^{1} \mathrm{H}$ NMR and were found negative to CPFAs. Each matrix was spiked with four different amounts of dihydrosterculic acid as reported in Experimental. Regression curves obtained are shown in Figure 4.

The linearity is maintained, as in the case of pure standard; however, in both cases, the intercept of the regression curve indicates a matrix effect, most pronounced for chicken fat. The limit of quantification for cheese was $120 \mathrm{mg} / \mathrm{kg}$ fat $(S / N$ ratio of 10$)$, and the limit of quantification was found to be $50 \mathrm{mg} / \mathrm{kg}$ fat $(S / N$ ratio of 10$)$. In the case of chicken meat, LOQ and LOD were $180 \mathrm{mg} / \mathrm{kg}$ fat and $70 \mathrm{mg} / \mathrm{kg}$ fat, respectively. Comparing these values with those obtained for pure standard solutions, results demonstrate not negligible matrix effect both in cheese and in 


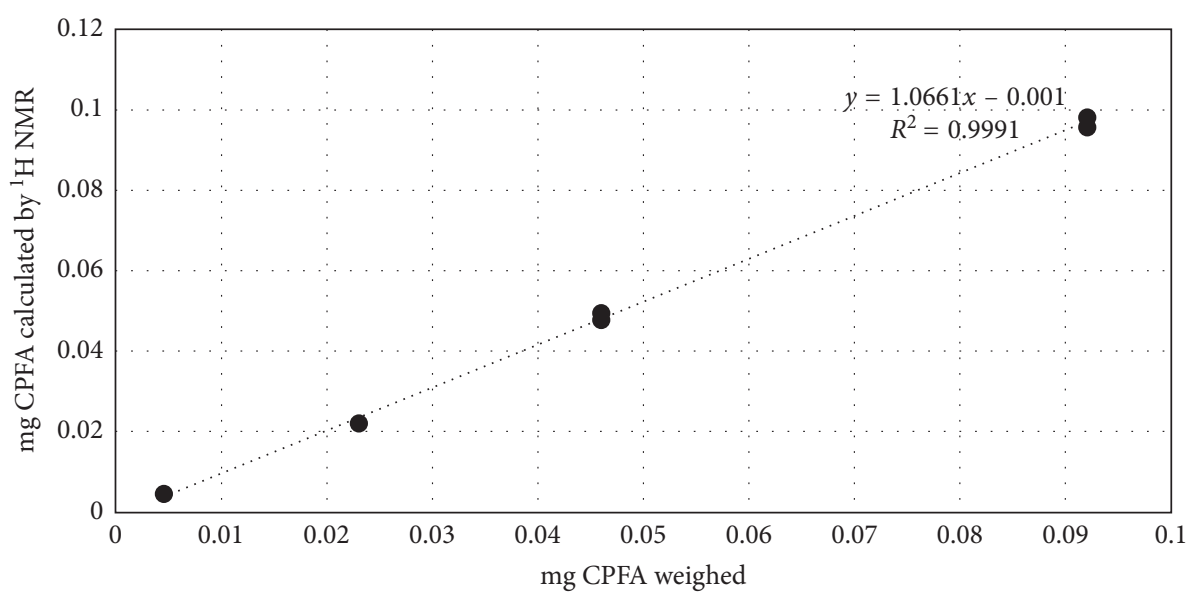

Figure 3: Regression curve for CPFA standard solution measured with the ${ }^{1} \mathrm{H}$ NMR method.

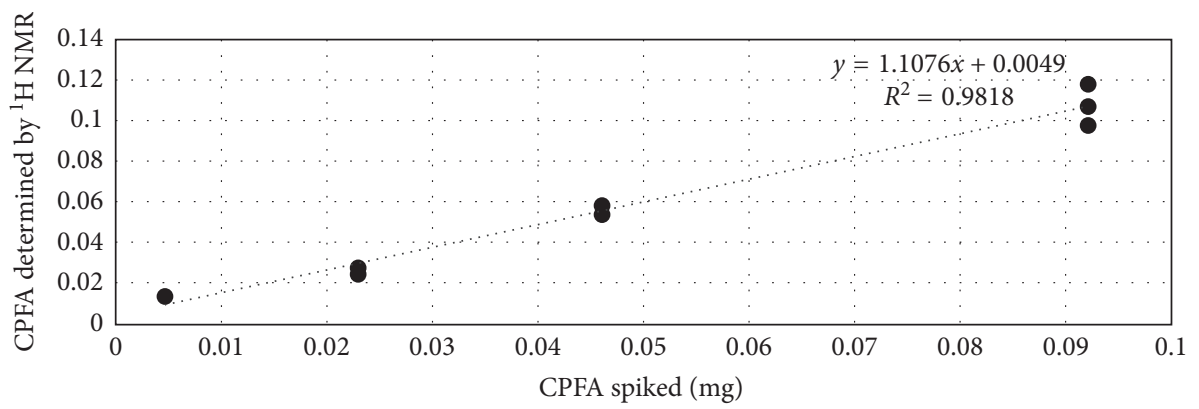

(a)

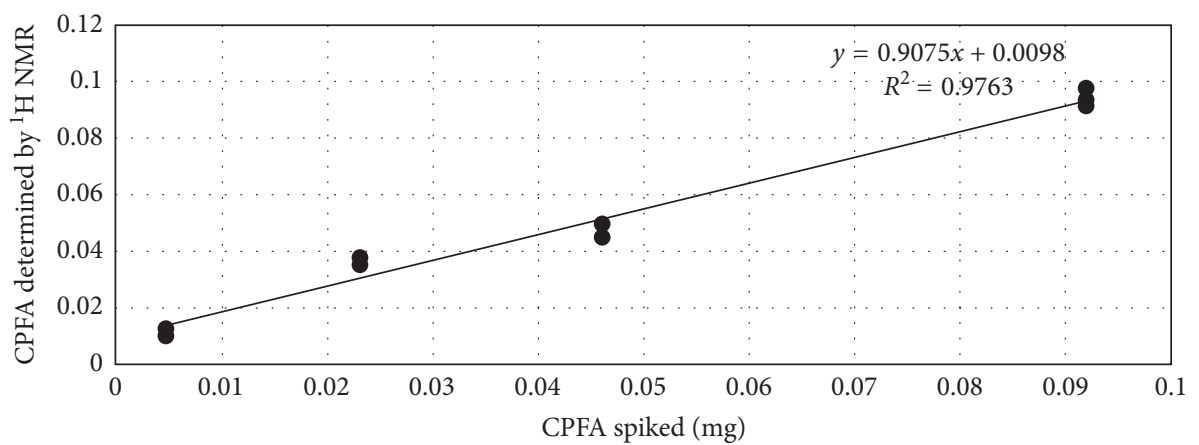

(b)

FIgURE 4: Calibration curves of CPFA spiked matrices: (a) cheese and (b) chicken meat.

meat fat, suggesting that an external calibration in a fat matrix is needed for an accurate quantification. On the contrary, the method can be easily applied for a rapid semiquantitative and qualitative analyses in both matrices.

\subsection{Application to Real Samples}

3.3.1. Comparison of ${ }^{1} \mathrm{H}$ NMR-Based Method and GC-MS Method. Different samples of meat, fish, and cheese were analysed both by ${ }^{1} \mathrm{H}$ NMR and GC-MS. GC-MS quantitative analysis was performed based on the method previously applied for cheese, as described in
Caligiani et al. [6]. Figure 5 shows the enlargement of the diagnostic region for CPFAs in the ${ }^{1} \mathrm{H}$ NMR spectra of lipids extracted from cheese, meat, and fish (containing the TMSD internal standard), confirming that the signal does not overlap with any other resonances representative of fatty acids.

Table 1 reports the list of the samples analysed for each food category, the number of samples negative or positive to CPFAs, and the comparison between GC-MS and ${ }^{1} \mathrm{H}$ NMR results. A reference Grana Padano cheese was specifically analysed both by GC-MS and ${ }^{1} \mathrm{H}$ NMR method to demonstrate the accuracy of the new ${ }^{1} \mathrm{H}$ NMR method. Then, five samples of Parmigiano Reggiano and five samples of Grana Padano were tested because in the case of cheese, we had 


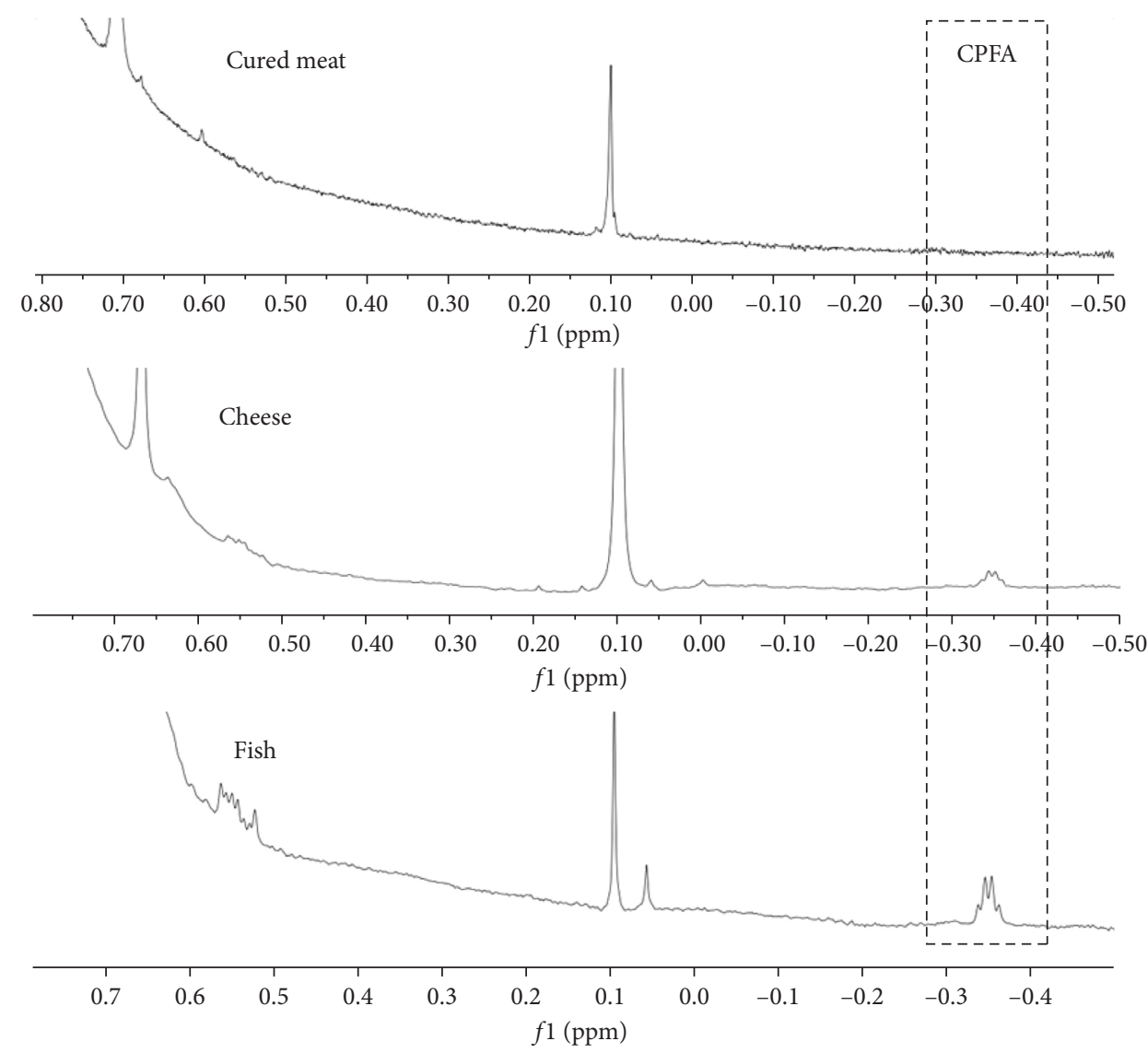

FIGURE 5: Enlargement of ${ }^{1} \mathrm{H}$ NMR $600 \mathrm{MHz}$ spectra in the zone from -0.5 to $0.8 \mathrm{ppm}$, showing the signal of the cyclopropane ring (at $-0.35 \mathrm{ppm}$ ) used for CPFA quantification in cured ham (negative to CPFAs) and cheese and fish fat (positive to CPFAs).

TABLE 1: Comparison of ${ }^{1} \mathrm{H}$ NMR and GC-MS results on the presence of CPFAs in some representative samples of fat of animal origin.

\begin{tabular}{|c|c|c|c|}
\hline Samples & $\begin{array}{c}\text { Number of analysed } \\
\text { samples }\end{array}$ & $\begin{array}{l}\text { CPFA (GC-MS) }(\mathrm{mg} / \mathrm{kg} \\
\text { fat) }\end{array}$ & $\begin{array}{c}\text { CPFA }\left({ }^{1} \mathrm{H} \text { NMR }\right)(\mathrm{mg} / \mathrm{kg} \\
\text { fat })\end{array}$ \\
\hline \multicolumn{4}{|l|}{ Cheese } \\
\hline Reference cheese & & $600 \pm 50$ & $690 \pm 60$ \\
\hline Parmigiano Reggiano & 5 & $<\mathrm{LOD}$ & $<\mathrm{LOD}$ \\
\hline Grana Padano & 5 & $400-700$ & $400-800$ \\
\hline \multicolumn{4}{|l|}{ Meat } \\
\hline Commercial bovine meat & 5 & $200-400$ & $300-400$ \\
\hline $\begin{array}{l}\text { Bovine meat of certified origin (not fed with } \\
\text { silages) }\end{array}$ & 2 & $<\mathrm{LOD}$ & $<\mathrm{LOD}$ \\
\hline Other meats (Pork and chicken) & 4 & $<\mathrm{LOD}$ & $<\mathrm{LOD}$ \\
\hline \multicolumn{4}{|l|}{ Cured meat } \\
\hline Salami & 1 & 60 & $<$ LOD \\
\hline Parma ham & 1 & 120 & $<\mathrm{LOD}$ \\
\hline Bresaola & 1 & 340 & 400 \\
\hline \multicolumn{4}{|l|}{ Fish } \\
\hline Eel & 2 & $180-350$ & $400-590$ \\
\hline Mullet & 1 & 120 & 800 \\
\hline
\end{tabular}

collected previously many data confirming the association between the use of ensiled feeds and the presence of CPFA $[4,6] .{ }^{1} \mathrm{H}$ NMR analysis confirmed the positivity at CPFA for all samples of Grana Padano (ensiled feeds allowed) and the negativity of all Parmigiano Reggiano samples (ensiled feeds forbidden), indicating that ${ }^{1} \mathrm{H}$ NMR could be an attractive alternative technique to GC-MS to assure the authenticity of Parmigiano Reggiano and other cheeses forbidding the use of ensiled feeds in their disciplinary of productions. In the case of cheese, quantitative results suggested very good agreement between data obtained by the quantitative ${ }^{1} \mathrm{H}$ NMR analysis and previous GC analysis method. 
Concerning meat, CPFAs were detected in the GC-MS profiles of most of the commercial bovine meat samples in concentrations varying from 100 to $400 \mathrm{mg} / \mathrm{kg}$ of the total fat. CPFAs were detected by ${ }^{1} \mathrm{H}$ NMR analysis in all commercial bovine meat samples previously resulted positive by the GC-MS analysis, with good agreement of the quantitative results. CPFAs were absent in two samples of certified meat from cows not fed with fermented forages, and this was evidenced by both techniques. The GC-MS analysis of other meat samples (pork and chicken) was negative to CPFAs both in GC-MS and ${ }^{1} \mathrm{H}$ NMR method. In the case of pork cured meat (salami and ham), the GC-MS analysis showed the presence of a signal at the retention time of CPFAs with concentrations of $60-100 \mathrm{mg} / \mathrm{kg}$ of the total fat. However, the corresponding analysis by ${ }^{1} \mathrm{H}$ NMR did not show the presence of cyclopropane ring, indicating the presence of an interfering peak in the GC-MS conditions adopted. This interfering peak was also resistant to oxidation as a saturated fatty acid, but it has not been identified yet and it was not easy to obtain a better separation varying chromatographic conditions. Therefore, in the case of pork, cured meat seems to be important to have the NMR confirmation of CPFA presence.

And in cured meat, the GC-MS analysis of fish samples generally showed the presence of interfering signals at the same retention time of cyclopropane fatty acids with the same corresponding mass spectrum $(278 \mathrm{~m} / z)$, probably due to the presence of different isomers of nonadecenoic acid. Moreover, lactobacillic acid coeluted with another interfering substance with the corresponding mass spectrum of $165 \mathrm{~m} / z$. This interfering peak was not resistant to oxidation and it has been suggested to be a furan fatty acid as discussed elsewhere [21, 22].

Therefore, GC-MS analysis alone was not able to confirm the presence or absence of CPFAs in fish samples, but it always required ${ }^{1} \mathrm{H}$ NMR analysis. Moreover, observing the preliminary results showed in Table 1 on three fish samples, it seems that GC-MS underestimates the content of cyclopropane fatty acids, suggesting that besides cyclopropane fatty acids with 19-carbon atom skeleton, such as dihydrosterculic and lactobacillic acids, it is possible that other CPFAs with different chain lengths occur in fish.

\section{Conclusion}

A new quantitative ${ }^{1} \mathrm{H}$ NMR method was developed for the determination of CPFA content in different food matrices, including dairy products, meat, and fish.

The new method reported here provides absolute quantities of CPFA (mg/kg of total fat) and shows a limit of detection comparable with those of full scan GC-MS. A complete and reliable sample analysis can be performed quickly and requires little sample preparation, reagents, and solvents. This was possible because the CPFA signal was very well defined and did not overlap with others. The role of NMR seems to be most important in meat and fish characterization because the GC-MS analysis was not able to confirm the presence of CPFAs in all the analysed samples due to the presence of interfering peaks.
Results suggested that the NMR analysis approach has potential application as a screening for quantifying cyclopropane fatty acids in meat and fish, as markers of quality and the preliminary data on few meat and fish samples presented here suggest some possible developments. For example, in the context of food authentication, cyclopropane fatty acids might be proposed, as in the case of cheese, as markers of silage feedings are able to authenticate highquality costly meat whose producers declare the absence of silages in the feeding. This will require the construction of a robust database of meat certificated for the feeding system. This approach could also be extended to fish, to eventually distinguish farmed from wild fish.

Moreover, in the case of fish, NMR method is able to detect a higher amount of CPFAs with respect to GC-MS, indicating an important role of NMR when dietary intake of cyclopropane fatty acid has to be assessed.

\section{Conflicts of Interest}

The authors declare that there are no conflicts of interest regarding the publication of this paper.

\section{References}

[1] C. Montanari, S. L. SadoKamdem, D. I. Serrazanetti, F. X. Etoa, and M. E. Guerzoni, "Synthesis of cyclopropane fatty acids in Lactobacillus helveticus and Lactobacillus sanfranciscensis and their cellular fatty acids changes following short term acid and cold stresses," Food Microbiology, vol. 27, no. 4, pp. 493-502, 2010.

[2] X. Bao, S. Katz, M. Pollard, and O. John, "Carbocyclic fatty acids in plants: biochemical and molecular genetic characterization of cyclopropane fatty acid synthesis of Sterculia foetida," Plant Biology, vol. 99, no. 10, pp. 7172-7177, 2002.

[3] A. Marseglia, A. Caligiani, L. Comino, F. Righi, A. Quarantelli, and G. Palla, "Cyclopropyl and $\omega$-cyclohexyl fatty acids as quality markers of cow milk and cheese," Food Chemistry, vol. 140, pp. 711-716, 2013.

[4] A. Caligiani, A. Marseglia, and G. Palla, "An overview on the presence of cyclopropane fatty acids in milk and dairy products," Journal of Agricultural and Food Chemistry, vol. 62, pp. 7828-7832, 2014.

[5] T. Sledzinski, A. Mika, P. Stepnowski et al., "Identification of cyclopropaneoctanoic acid 2-hexyl in human adipose tissue and serum," Lipids, vol. 48, no. 8, pp. 839-848, 2013.

[6] A. Caligiani, M. Nocetti, V. Lolli, A. Marseglia, and G. Palla, "Development of a quantitative GC-MS method for the detection of cyclopropane fatty acids in cheese as new molecular markers for Parmigiano Reggiano authentication," Journal of Agricultural and Food Chemistry, vol. 64, pp. 4158-4164, 2016.

[7] W. W. Christie, Gas Chromatography and Lipids: A Practical Guide, The Oily Press, Bridgwater, UK, 1989.

[8] P. Delmonte, A. R. Fardin-Kia, J. K. Kramer et al., "Evaluation of highly polar ionic liquid gas chromatographic column for the determination of the fatty acids in milk fat," Journal of Chromatography A, vol. 1233, pp. 137-146, 2012.

[9] J. Ecker, M. Scherer, G. Schmitz, and G. Liebisch, "A rapid GC-MS method for quantification of positional and geometric isomers of fatty acid methyl esters," Journal of Chromatography B, vol. 897, pp. 98-104, 2012. 
[10] D. Prema, T. D. Turner, J. Jensen et al., "Rapid determination of total conjugated linoleic acid concentrations in beef by ${ }^{1} \mathrm{H}$ NMR spectroscopy," Journal of Food Composition and Analysis, vol. 41, pp. 54-57, 2015.

[11] M. Schröder and W. Vetter, "Detection of 430 fatty acid methyl esters from a transesterified butter sample," Journal of the American Oil Chemical Society, vol. 90, p. 771, 2013.

[12] G. Le Gall and I. J. Colquhoun, "NMR spectroscopy in food authentication," in Food Authenticity and Traceability, M. Lees, Ed., pp. 131-155, Woodhead Publishing Ltd, Cambridge, UK, 2003.

[13] A. Barison, C. W. Pereira da Silva, F. R. Campos, F. Simonelli, C. A. Lenz, and A. G. Ferreira, "A simple methodology for the determination of fatty acid composition in edible oils through ${ }^{1} \mathrm{H}$ NMR spectroscopy," Magnetic Resonance in Chemistry, vol. 48 , pp. 642-650, 2010.

[14] W. Jakes, A. Gerdova, M. Defernez et al., "Authentication of beef versus horse meat using $60 \mathrm{MHz}{ }^{1} \mathrm{H}$ NMR spectroscopy," Food Chemistry, vol. 175, pp. 1-9, 2015.

[15] R. M. Maria, L. A. Colnago, L. A. Forato, and D. Bouchard, "Fast and simple nuclear magnetic resonance method to measure conjugated linoleic acid in beef," Journal of Agricultural and Food Chemistry, vol. 58, pp. 6562-6564, 2010.

[16] G. Knothe, "NMR characterization of dihydrosterculic acid and its methyl ester," Lipids, vol. 41, no. 4, 2006.

[17] M. Baranac-Stojanovic and M. Stojanovic, " ${ }^{1} \mathrm{H}$ NMR chemical shifts of cyclopropane and cyclobutane: a theoretical study," Journal of Organic Chemistry, vol. 78, pp. 1504-1507, 2013.

[18] J. Folch, M. Less, and G. H. Sloane, "A simple method for the isolation and purification of total lipids from animal tissues," Journal of Biology and Chemistry, vol. 226, pp. 497-509, 1957.

[19] J. Müller Maatsch, A. Caligiani, T. Tedeschi, K. Elst, and S. Sforza, "Simple and validated quantitative ${ }^{1} \mathrm{H}$ NMR method for the determination of methylation, acetylation, and feruloylation degree of pectin," Journal of Agricultural and Food Chemistry, vol. 62, pp. 9081-9087, 2014.

[20] I. Apostol, K. J. Miller, J. Ratto, and D. N. Kelner, "Comparison of different approaches for evaluation of the detection and quantitation limits of a purity method: a case study using a capillary isoelectrofocusing method for a monoclonal antibody," Analytical Biochemistry, vol. 385, pp. 101-106, 2009.

[21] G. Spiteller, "Furan fatty acids: occurrence, synthesis, and reactions. Are furan fatty acids responsible for the cardioprotective effects of fish diet?," Lipids, vol. 40, pp. 755-771, 2005.

[22] C. Truzzi, S. Illuminati, A. Annibaldi, M. Antonucci, and G. Scarponi, "Quantification of fatty acids in the muscle of Antarctic fish Trematomus bernacchii by gas chromatographymass spectrometry: optimization of the analytical methodology," Chemosphere, vol. 173, pp. 116-123, 2017. 

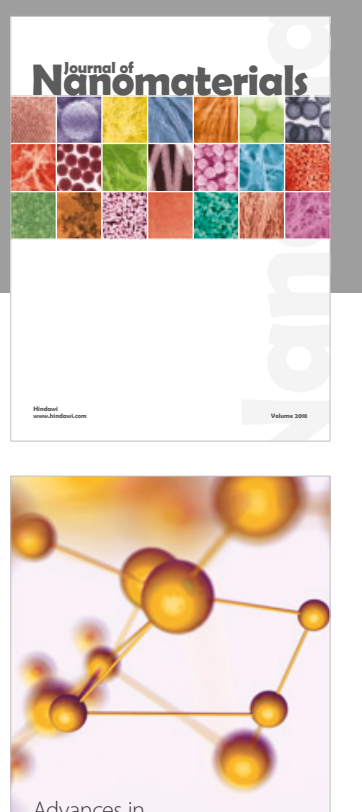

Physical Chemistry
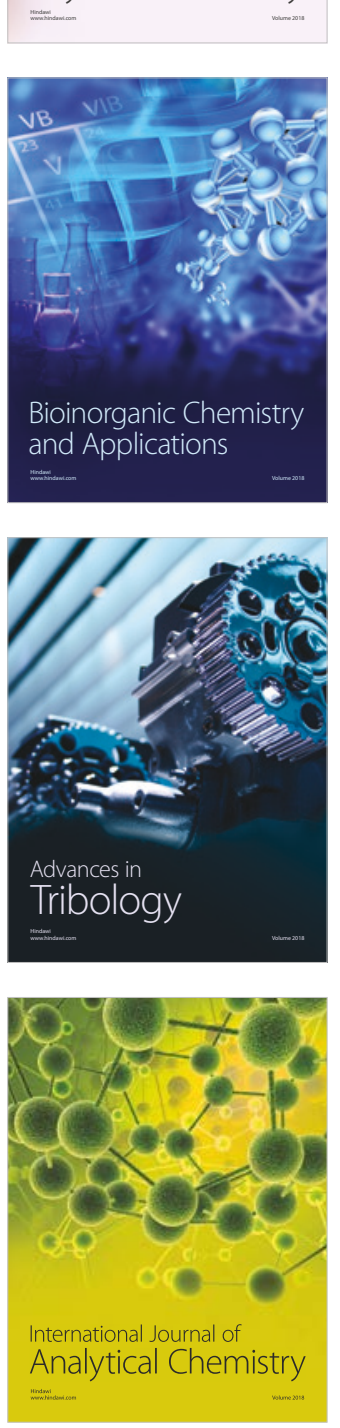

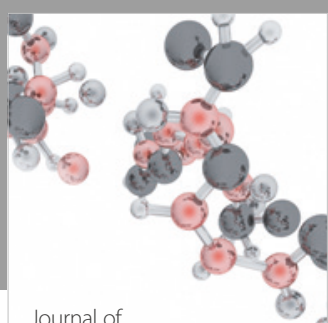

Analytical Methods

in Chemistry

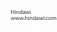

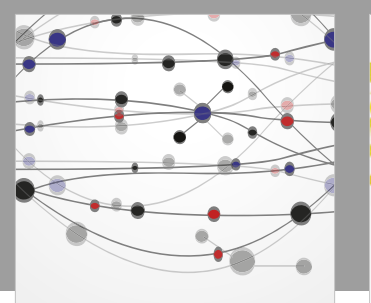

The Scientific World Journal

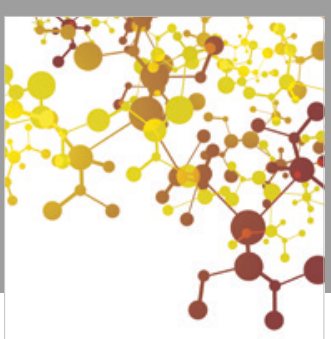

Journal of

Applied Chemistry
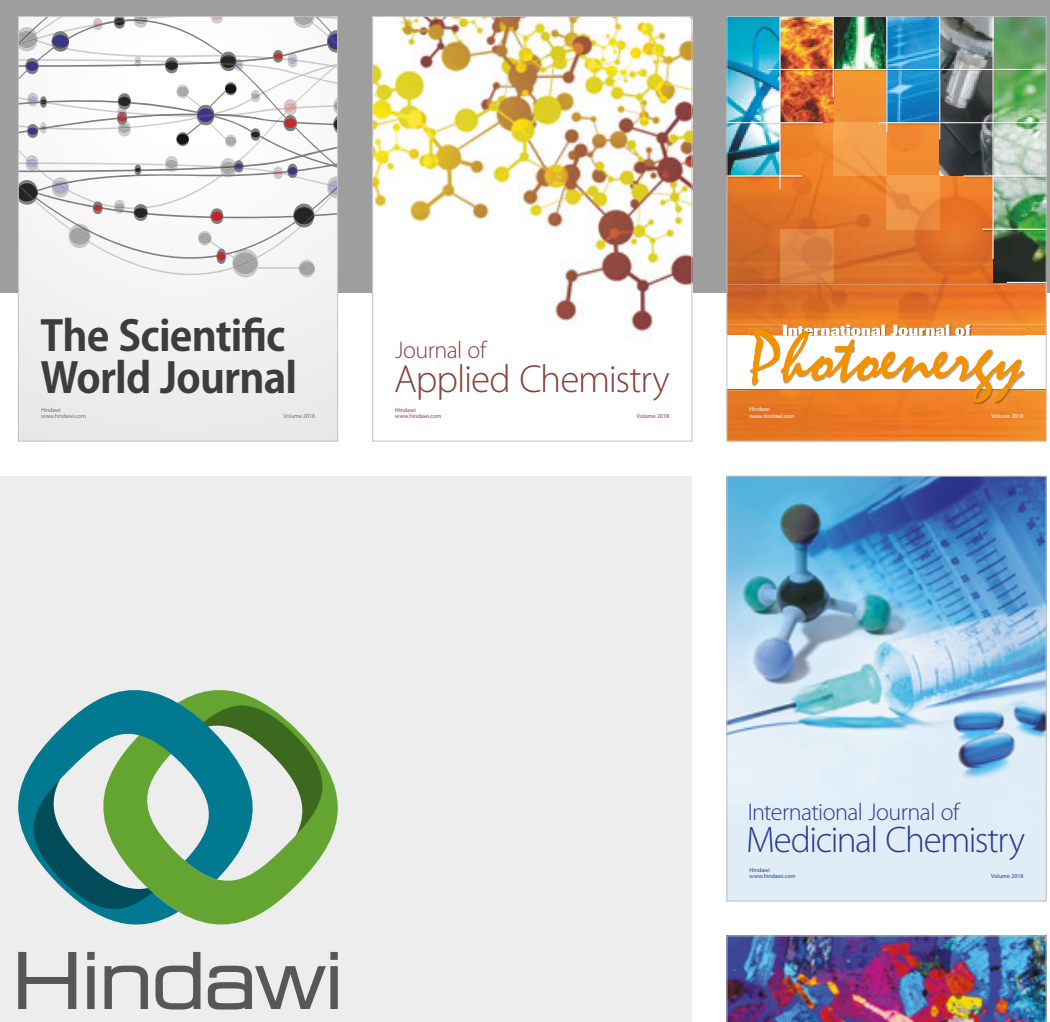

Submit your manuscripts at

www.hindawi.com
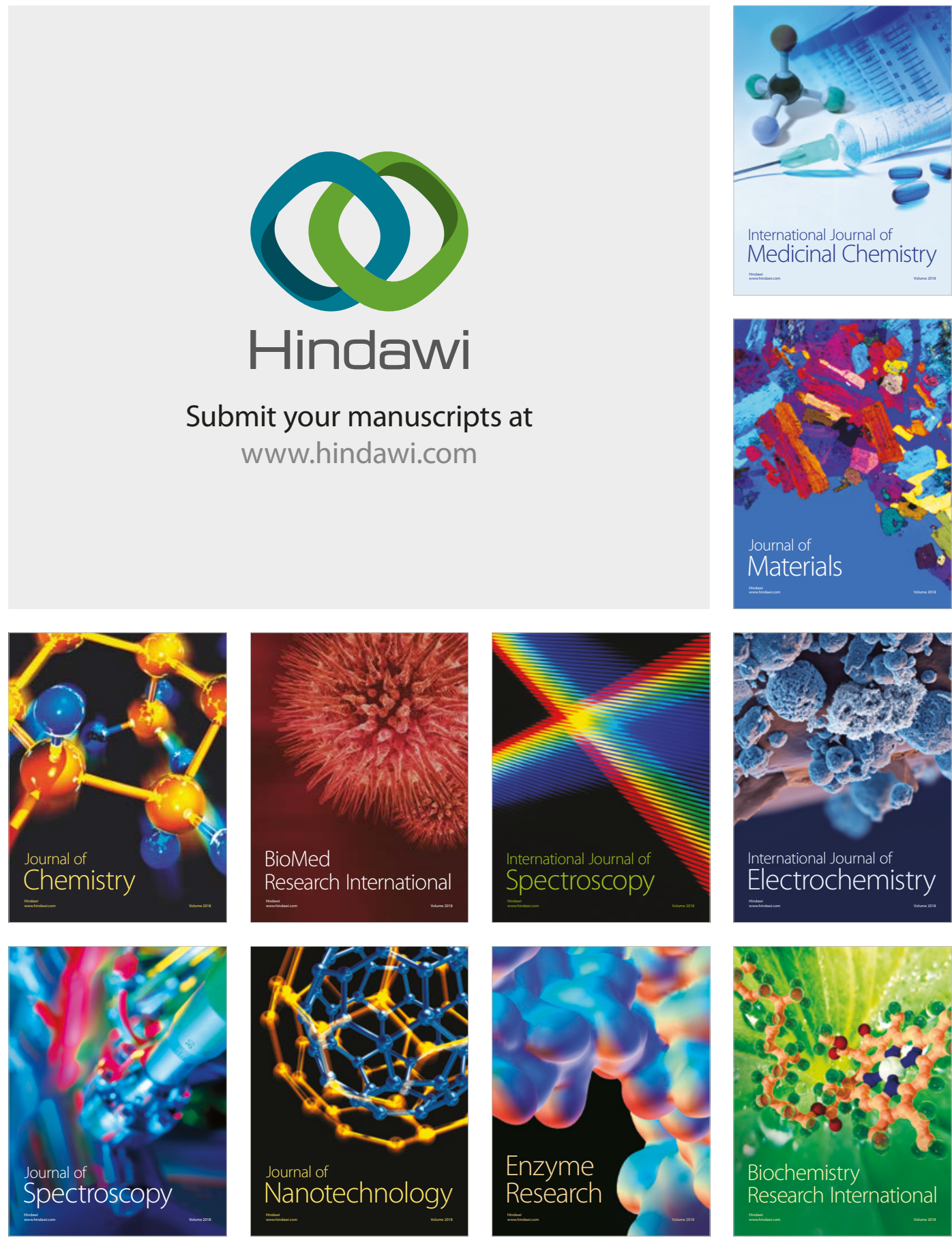
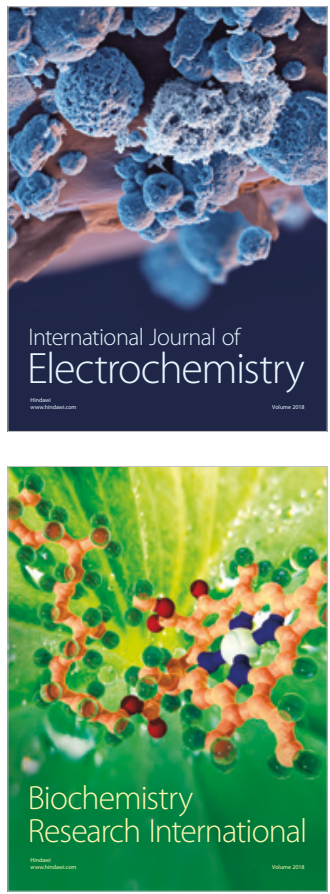